DRAFT VERSION SEPTEMBER 12, 2018

Preprint typeset using LTEX style emulateapj v. 5/2/11

\title{
PROBING THE ENVIRONMENT OF GRAVITATIONAL WAVE TRANSIENT SOURCES WITH TEV AFTERGLOW EMISSION
}

\author{
QIN-YU ZHU, XIANG-YU WANG ${ }^{1,2}$ \\ ${ }^{1}$ School of Astronomy and Space Science, Nanjing University, Nanjing 210093, China \\ ${ }^{2}$ Key laboratory of Modern Astronomy and Astrophysics (Nanjing University), Ministry of Education, Nanjing 210093, China
}

Draft version September 12, 2018

\begin{abstract}
Recently, Advanced Laser Interferometer Gravitational-wave Observatory (aLIGO) detected gravitational wave (GW) transients from mergers of binary black holes (BHs). The system may also produce a wide-angle, relativistic outflow if the claimed short GRB detected by GBM is in real association with GW 150914. It was suggested that mergers of double neutron stars (or neutron star-black hole binaries), another promising source of GW transients, also produce fast, wide-angle outflows. In this paper, we calculate the high-energy gamma-ray emission arising from the blast waves driven by these wide-angle outflows. We find that TeV emission arising from the inverse-Compton process in the relativistic outflow resulted from mergers of binary BHs similar to those in GW 150914 could be detectable by ground-based IACT telescopes such as Cherenkov Telescope Array (CTA) if the sources occur in a dense medium with density $n \gtrsim 0.3 \mathrm{~cm}^{-3}$. For neutron starneutron star (NS-NS) and NS-BH mergers, TeV emission from the wide-angle, mildly-relativistic outflow could be detected as well if they occur in a dense medium with $n \gtrsim 10-100 \mathrm{~cm}^{-3}$. Thus TeV afterglow emission would be a useful probe of the environment of the GW transients, which could shed light on the evolution channels of the progenitors of GW transients.
\end{abstract}

Subject headings: gamma-rays- gravitational waves

\section{INTRODUCTION}

The era of gravitational wave astronomy has begun with the first science run of the recently upgraded LIGO from 2015 September to 2016 January (Abbott et al. 2016a). The GW frequency range that LIGO and Virgo are sensitive to is expected to be dominated by mergers of compact stellar-mass objects that are most likely remnants of stellar evolution: two neutron stars (NS-NS), two black holes (BH-BH), or a NS and a BH. On 2015 September 14 at 09:50:45 UTC the LIGO collaboration detected the first GW event-GW150914. The trigger was determined to be consistent with a waveform predicted by General Relativity from the inspiral and merger of two stellar-mass BHs, with masses around $29 M_{\odot}$ and $36 M_{\odot}$, respectively (Abbott et al. 2016a).

Connaughton et al. (2016) reported a tentative detection of a weak transient gamma-ray source GW150914-GBM lasting about $1 \mathrm{~s}, 0.4 \mathrm{~s}$ after the LIGO trigger on GW150914. GW150914-GBM is consistent with being due to a lowfluence short gamma-ray burst (GRB) at an unfavorable viewing geometry to the GBM detectors, which is not expected from a $\mathrm{BH}-\mathrm{BH}$ merger. The isotropic-equivalent luminosity of GW150914-GBM in the $1 \mathrm{keV}$ to $10 \mathrm{MeV}$ energy range was $1.8_{-1.0}^{+1.5} \times 10^{49} \mathrm{ergs}^{-1}$ using a $410_{-180}^{+160} \mathrm{Mpc}$ distance inferred from the GW150914 event. GW150914 was outside of the field of view of the Fermi Large Area Telescope (LAT) initially and no GeV afterglow was detected when it could observe (Ackermann et al. 2016).

As the ground-based Imaging Atmospheric Cherenkov Telescopes (IACTs) usually have a better sensitivity than Fermi/LAT, we here study whether the $\mathrm{TeV}$ emission from the GW events could be detected by future IACTs, such as the planned Cherenkov Telescope Array (CTA). It has been argued that CTA is well suited to follow up GW transients (Bartos et al. 2014). The short GRB possibly associated with
GW150914 should be produced by a wide-angle outflow, since the chance of detection would be too low if it is a strongly beamed jet. The outflow producing this short GRB must be highly relativistic. It has been suggested that an accretion disk may exist around one of the BHs via the formation of the dead zone (Perna et al. 2014) in a BH-BH merger system. This system could then also have sub-relativistic or mildly relativistic wind outflow if the disk produce a wind outflow (Murase et al. 2016).

For the NS-NS mergers or NS-BH merges, significant wideangle mass outflows are also expected, as have been seen in the numerical simulations. The mass of the outflow is in the range of $M_{e j}=10^{-4}-10^{-2} M_{\odot}$ with a velocity of $v_{w}=$ $0.1-0.3 c$. For NS-BH mergers, the ejecta mass can be up to $\sim 0.1 M_{\odot}$ (Kyutoku et al. 2015). A fraction of the mass may have a higher speed or become relativistic with a Lorentz factor $\Gamma_{0} \sim 2$ and energy about $\sim 10^{49} \mathrm{erg}$ for NS-NS mergers or $\sim 10^{50} \mathrm{erg}$ for NS-BH mergers (Nakar \& Piran 2011). In this paper, we calculate the expected high-energy gammaray emission produced by the blast waves driven by these wide-angle outflows. The high-energy gamma-ray emission from the small-angle, relativistic jets has been calculated in literatures (Veres \& Mészáros 2014; Bartos et al. 2014). We here study the $\mathrm{TeV}$ emission from the wide-angle, mildlyrelativistic outflows. Compared with the small-angle jets, the wide-angle geometry can significantly increase the probability for the follow-up electromagnetic detections of GW transients.

We present the calculation of the self inverse-Compton (IC) emission for both relativistic and mildly-relativistic outflows in $\S 2$. In $\S 3$, we apply the result to both the BH-BH merger and NS-NS (and NS-BH) merger scenarios. Finally, we give our discussions and conclusions.

\section{THE IC EMISSION FROM WIDE-ANGLE OUTFLOWS}




\subsection{The IC emission from the blast wave}

The short GRB possibly associated with GW150914, if real, should be produced by a highly relativistic, wide-angle outflow. The calculation for relativistic wide-angle outflow is quite similar to the case of GRB jets except that the outflow may have a much wider opening angle. We assume the relativistic outflow has an initial Lorentz factor $\Gamma_{0} \sim 100$ and it interacts with homogenous ambient medium with a number density of $n$. Then the blast wave driven by the outflow starts to decelerate at a time $t_{d e c}=\left(E /\left(32 \pi n m_{p} \Gamma_{0}^{8} c^{5}\right)\right)^{1 / 3}=$ $10 \mathrm{~s} E_{50}^{1 / 3} n^{-1 / 3} \Gamma_{0,2}^{-8 / 3}$ and becomes non-relativistic at a time $t_{n r}=$ $\left(3 E /\left(4 \pi n m_{p} c^{2}\right)\right)^{1 / 3}=65 E_{50}^{1 / 3} n^{-1 / 3}$ days, where $E$ is the kinetic energy of the blast wave.

For the NS-NS/NS-BH mergers, numerical simulations show wide-angle outflows with mass $m=0.01-0.1 M_{\odot}$ and typical velocity $v=0.1-0.5 c$. Some works (e.g., Levinson 2006; Metzger et al. 2008; Dessart et al. 2009 ) suggest the inner part of the outflow can be accelerated up to relativistic velocities if the compact binaries form an accretion disk before the coalescence. We assume that the mildly-relativistic ejecta with $\Gamma \simeq 2$ have an energy of about $10^{49}-10^{50} \mathrm{erg}$. Such amount of energy is consistent with the non-detection of the late-time radio emission in some compact binary mergers (Horesh et al. 2016). For the mildly-relativistic outflow, the peak of the IC emission occurs at the deceleration time, which is $t_{d e c} \simeq 4.3 \times 10^{5} \mathrm{~s} E_{50}^{1 / 3} n^{-1 / 3}\left(\Gamma_{0} / 2\right)^{-8 / 3}$.

$\mathrm{TeV}$ emission is mainly produced by synchrotron selfCompton (SSC) process of shock-accelerated electrons. The typical spectral breaks in the SSC spectrum are (Sari \& Esin 2001)

$$
\nu_{m}^{I C}=2 \gamma_{m}^{2} \nu_{m}=3.36 \times 10^{14} \mathrm{~Hz} f_{p}^{4} \epsilon_{e, 0}^{4} \epsilon_{B,-2}^{1 / 2} E_{50}^{3 / 4} n^{-1 / 4} t_{6}^{-9 / 4}
$$

and

$$
\nu_{c}^{I C}=2 \gamma_{c}^{2} \nu_{c}=6.03 \times 10^{26} \mathrm{~Hz}_{B,-2}^{-7 / 2} t_{6}^{-1 / 4} E_{50}^{-5 / 4} n^{-9 / 4},
$$

where $p$ is the power-law index of the energy distribution of the injected electrons, $f_{p}=6(p-2) /(p-1), \epsilon_{e}$ and $\epsilon_{B}$ are respectively the equipartition factors of magnetic field and electron energy, $\gamma_{m}=\epsilon_{e} \frac{p-2}{p-1} \frac{m_{p}}{m_{e}}(\Gamma-1)$ and $\gamma_{c}$ are two characteristic Lorentz factors in the electron energy distribution, and $\nu_{m}$ and $\nu_{c}$ are the corresponding break frequencies (Sari et al. 1998). The optical depth for IC scatterings is $\tau=\frac{1}{3} \sigma_{\mathrm{T}} n R$ for a constant density medium, where $R$ is the radius of the blast wave. Then one can obtain the peak flux for the SSC component,

$$
f_{m}^{I C}=\tau f_{m}=1.08 \times 10^{-7} \mathrm{mJy}_{50}^{5 / 4} n^{5 / 4} \epsilon_{B,-2}^{1 / 2} t_{6}^{1 / 4} D_{L, 27}^{-2},
$$

where $f_{m}$ is the peak flux of the synchrotron component and $D_{L}$ is the luminosity distance of the source. The flux of the IC component is given by

$$
f_{\nu}=\left\{\begin{array}{l}
f_{m}^{I C}\left(\frac{\nu}{\nu_{m}^{I C}}\right)^{-\frac{p-1}{2}} \quad, \nu_{m}^{I C}<\nu<\nu_{c}^{I C} \\
f_{m}^{I C}\left(\frac{\nu_{c}^{I C}}{\nu_{m}^{I C}}\right)^{-\frac{p-1}{2}}\left(\frac{\nu}{\nu_{c}^{I C}}\right)^{-\frac{p}{2}} \quad, \nu_{c}^{I C}<\nu .
\end{array}\right.
$$

At high energies, the SSC spectrum could be affected by the Klein-Nishina effect (Nakar et al. 2009; Wang et al. 2010), which occurs at frequencies above a critic frequency of

$$
\nu_{c}^{K N}=\gamma_{c} m_{e} c^{2}=2.4 \times 10^{25} \mathrm{~Hz} \epsilon_{B,-2}^{-1} E_{50}^{-3 / 8} n^{-5 / 8} t_{6}^{1 / 8} .
$$

Then the flux can be obtained by using Eq.50 in Nakar et al. (2009).
To illustrate the TeV afterglow behavior, we show an example of the light curves at $h \nu=1 \mathrm{TeV}$ by taking some reference values for the parameters of the wide-angle outflows, which is shown in Fig.1. The relativistic wide-angle outflow generates a decaying TeV afterglow. Meanwhile, the mildlyrelativistic outflow and sub-relativistic outflow produce a rising light curve before the deceleration time and a decay one after that. Before deceleration, since the blast wave velocity is constant, the typical energy of electrons $\left(\gamma_{m}\right)$ and the peak frequency $\left(\nu_{m}^{I C}\right)$ are constant. As the number of radiating electrons is increasing with time, the inverse-Compton TeV flux increases with time. After deceleration, as the blast wave velocity decreases, the power of the inverse-Compton emission per electron decreases rapidly with time, so the $\mathrm{TeV}$ flux starts to decrease with time.

Our calculations are based on the assumption of the electron distribution of $p=2.2$. A larger $p$ may suppress the high energy gamma-ray emission. This is because the $\mathrm{TeV}$ band is much larger than the peak frequency of SSC emission $v_{m}^{I C}$ and even larger than the cooling frequency $v_{c}^{I C}$ in some cases, which makes the ratio between the TeV flux and the peak SSC flux affected by the electron distribution.

\subsection{Detectability by CTA}

We first need to estimate the sensitivity of CTA. According to the performance of CTA $\square$, CTA South has a differential sensitivity of $E^{2} \phi_{0}=1 \times 10^{-13} \mathrm{erg} \mathrm{cm}^{-2} \mathrm{~s}^{-1}$ at energy of $1 \mathrm{TeV}$ for the $T_{0}=50 \mathrm{~h}$ exposure. Following the method in Gou \& Mészáros (2007), the sensitivity for an exposure time $t_{\text {exp }}$ can be determined by the minimum fluence $F\left(t_{\text {exp }}\right)$ with which a source is detecable (Bartos et al. 2014), i.e. $F\left(t_{\text {exp }}\right) \approx \kappa \phi_{0}\left(\frac{T_{0}}{t_{\text {exp }}}\right)^{1 / 2} E_{\text {cut }}^{2} t_{\text {exp }}$, where $E_{\text {cut }}$ is the cutoff energy of the energy spectrum, and the constant $\kappa \leq 1$ depends on the width of the energy bin, the flux from the source and the spectral shape of the detector sensitivity. Hereafter, we conservatively assume $\kappa=1$.

To determine the direction of a GW event, at least two separate $\mathrm{GW}$ detectors are needed, taking advantage of the different arrival time of the GW signals at different locations (Abbott et al. 2016d and references therein). Based on the results of Fairhurst (2011), Bartos et al. (2014) estimated the typical localization sky area for LIGO Hanford and LIGO Livingstone network to be $\Omega \approx 2000 \operatorname{deg}^{2}\left(\frac{8}{\operatorname{SNR}}\right)\left(\frac{\operatorname{erf}^{-1}(C L)}{\operatorname{erf}^{-1}(0.9)}\right)$ where $\operatorname{erf}^{-1}(C L)$ is the inverse error function at certain confidence level (CL) and we normally choose a confidence level of $0.9(90 \%)$. For the three detector case, the localization sky area could be reduced to $\Omega \approx 100 \operatorname{deg}^{2}\left(\frac{8}{\mathrm{SNR}^{2}}\right)\left(\frac{\mathrm{erf}^{-1}(\mathrm{CL})}{\mathrm{erf}^{-1}(0.9)}\right)$ or even better $\left(<20 \mathrm{deg}^{2}\right)$ at some special locations. For the medium energy range of CTA, from $100 \mathrm{GeV}$ to $10 \mathrm{TeV}$, CTA has 40 telescopes with a Field-of-View of about $38 \mathrm{deg}^{2}$. Therefore, CTA can cover the required area determined by three (or more) GW detectors with only 1-3 follow-up observation(s). According to Bartos et al. (2014), we find that the sensitivity in the survey mode is $S_{\text {survey }} \geq 0.5 S_{d e t}$, where $S_{\text {det }}=F\left(t_{\text {exp }}\right) / t_{\text {exp }}$ is the single-pointing detection threshold of CTA. Hereafter, we use $S_{d e t}$ as the TeV sensitivity of CTA approximately.

As CTA can slew to a certain position within 100 seconds, it can observe the early stage of the TeV afterglow if the GW transient is localized, for example, by a short GRB. For the

\footnotetext{
${ }^{1}$ https://portal.cta-observatory.org/Pages/CTA-Performance.aspx
} 
mildly-relativistic outflow case, even if the spatial error is as large as $100 \mathrm{deg}^{2}$, CTA can cover the error in the survey model with a few consecutive observations. The sensitivity light curve of CTA in the point observation mode is shown in Fig.1. It is worth noting that the CTA limit observation time is $t_{o b} \leq 50 \mathrm{~h}$ because of observing strategies, so we fix the sensitivity when $t>50 \mathrm{~h}$.

\section{TEV AFTERGLOWS IN SPECIFIC MODELS}

\section{1. $\mathrm{BH}-\mathrm{BH}$ mergers}

The possible short GRB associated with GW150914 suggests that $\mathrm{BH}-\mathrm{BH}$ mergers may produce wide-angle, relativistic outflows with an isotropic energy of $E \sim 10^{50} \mathrm{erg}$ (Connaughton et al. 2016). We study the conditions under which the $\mathrm{TeV}$ afterglow from this relativistic outflow can be detected by CTA. Fig.2 shows the TeV flux as a function of the density $n$ and the magnetic field equipartition factor $\epsilon_{B}$, as these two parameters are the mostly unknown parameters. The distance of the source is taken as $D_{L}=10^{27} \mathrm{~cm}$, comparable to the distance of GW150914 (Abbott et al. 2016a). Since the $\mathrm{TeV}$ light curves show a decaying feature (Fig.1), the time at which CTA starts observation, $t_{\text {start }}$, is important for $\mathrm{TeV}$ detection. A conservative observational delay of $t_{\text {start }} \geq 100 \mathrm{~s}$ can be obtained because of a 1-minute delay from data analysis/detection and 1/2 minute delay from the maneuver of CTA (Bartos et al. 2014). We show how the value of $t_{\text {start }}$ affects CTA detection by choosing two values of $10^{3} \mathrm{~s}$ and $10^{4} \mathrm{~s}$ respectively, as shown respectively by the left and right panel of Fig.2. We find that the TeV afterglow emission for the case of $t_{\text {start }}=10^{4} \mathrm{~s}$ can be detected by CTA when $n \gtrsim 1 \mathrm{~cm}^{-3}$ for $\epsilon_{B} \gtrsim 10^{-3}$, while for the case of $t_{\text {start }}=10^{3} \mathrm{~s}, n \gtrsim 0.3 \mathrm{~cm}^{-3}$ is needed.

\subsection{NS-NS / NS-BH mergers}

The mergers of NS-NS or NS-BH binaries are promising sources for gravitational detection by aLIGO and aVirgo. Short GRBs, resulted from highly relativistic jets, are believed to be produced by such systems. As the opening angle of the relativistic jets are usually small, perhaps of $\theta_{j} \sim 0.1$ (Berger 2014), the jet direction only covers a very small fraction of the sky. Here we pay attention to the wide-angle outflow from NS-NS (NS-BH) mergers. Numerical simulations of compact binary merger systems have been carried out by many groups (e.g. Rosswog et al. 1999, 2000; Rosswog 2005; Ruffert \& Janka 2001; Yamamoto et al. 2008; Rezzolla et al. 2010; Kiuchi et al. 2010). The mergers give rise to unbound matter ejection through dynamic processes. The mass ejected depends primarily on the total binary mass, the mass ratio and the equation of state. In some simulations, disk formation can contribute to several outflow sources. For example, neutrino heating drives a wind from the disk surface (e.g., Levinson 2006; Metzger et al. 2008; Dessart et al. 2009). The outflow velocity may become relativistic for winds that are ejected from close to the central object. Thus, similar to Nakar \& Piran (2011), we assume that a fraction of the mass becomes relativistic with a Lorentz factor of $\Gamma \sim 2$ and energy about $\sim 10^{49} \mathrm{erg}$ for NS-NS mergers or $\sim 10^{50} \mathrm{erg}$ for NS-BH mergers. If the central object after the merger of double NSs is a fast-rotating Magnetar, the energy in the mildly-relativistic ejecta would be even larger 2 (Thompson et al. 2004).

\footnotetext{
${ }^{2}$ Wang et al. (2016) have studied the SSC emission from the reverse shock powered by post-merger millisecond magnetars.
}

We calculate the $\mathrm{TeV}$ afterglow emission at the deceleration time of the mildly-relativistic outflow, which is shown in Fig.3. In this case, the light curve reaches a peak at the time $t_{\text {dec }} \sim 3.0 \times 10^{5} E_{49}^{1 / 3} n^{-1 / 3} \mathrm{~s}$. The duration of the peak lasts several days, so CTA can scan the whole error box of the GW transient in this period. According to Fig.3, the detection of a NS-BH merger at $D_{L}=10^{26.5} \mathrm{~cm}$ by CTA requires that the density should be $n \gtrsim 10 \mathrm{~cm}^{-3}$ for a wide range of $\epsilon_{B}\left(10^{-4} \lesssim \epsilon_{B} \lesssim 10^{-2}\right)$. Such a high density is possible if the merger occurs in the disk of starburst galaxies. We note that some short GRBs have been found to occur in such starforming galaxies (Berger 2014). Detection of $\mathrm{TeV}$ afterglow would strongly support that mergers indeed occur in the starforming regions.

\section{CONCLUSIONS AND DISCUSSIONS}

We considered the $\mathrm{TeV}$ afterglow emission from the wideangle, relativistic outflow of BH-BH mergers and from the mildly-relativistic outflow of NS-NS/NS-BH mergers. We found that $\mathrm{TeV}$ afterglow emission could be detected if the compact binary mergers occur in a dense ambient environment(i.e., $n \gtrsim 0.3 \mathrm{~cm}^{-3}$ for GW150914-like binary BH mergers and $n \gtrsim 10-100 \mathrm{~cm}^{-3}$ for NS-NS/NS-BH mergers). For the binary black-hole merger GW150914, it has been suggested that the binary black-holes formation involves either isolated binaries in galactic fields or dynamical interactions in young and old dense stellar environments Abbott et al. 2016c). The binary black-hole progenitor of GW150914 could have formed in the local universe with a short merger delay time, or it could have formed at a higher redshift with a long merger delay time. In the short-delay merger scenario, the mergers are expected to occur in the star-forming regions, so the ambient medium density is high. Detections of $\mathrm{TeV}$ afterglow emission by CTA in future would support the shortdelay merger scenario for GW events like GW150914.

For the evolution of NS-NS or NS-BH binaries, short GRB observations have shed some light on the burst environment. Short GRBs occur in both elliptical and star-forming galaxies, with the latter accounting for about $80 \%$ of the sample (Berger 2014). For mergers occurring in dense star-forming regions with a high gas density, $\mathrm{TeV}$ afterglow emission could be detected by CTA. If a Magnetar forms after the merger of double NSs, the chance of detection of $\mathrm{TeV}$ afterglow would be increased as the energy in the mildly-relativistic outflow is increased by the injection energy from the central Magnetar.

The mildly-relativistic ejecta resulted from NS-NS or NSBH mergers will also produce a long term radio emission due to its interaction with the surrounding ISM. Recently, Horesh et al. (2016) search for such emission from two short GRBs, i.e. GRB 130603B and GRB 060614, with radio telescopes, but find non-detections. The upper limit flux of the radio emission can nevertheless put useful constraints on the parameters of the ejecta and density of the surrounding medium. To study the constraints, we calculate the radio emissions at $6.7 \mathrm{GHz}$ from the mildly-relativistic ejecta with different parameters adopted, which are shown in Fig.4. We find that the radio emission violates the upper limits only when both the ISM density is higher than $\sim 100 \mathrm{~cm}^{-3}$ and the ejecta energy is higher than $10^{50} \mathrm{erg}$. Therefore, a wide parameter space still exists for which $\mathrm{TeV}$ afterglow could be detected by CTA while meanwhile the radio constraints are satisfied.

We thank Liang-Duan Liu and Yuan-Pei Yang for helpful 
discussions. This work is supported by the National Basic Research Program (973 Program) of China under Grant No. 2014CB845800, the National Natural Science Foundation of
China under Grants No. 11273016, and the Basic Research Program of Jiangsu Province under Grant No. BK2012011.

\section{REFERENCES}

Ackermann, M., Ajello, M., Albert, A., et al. 2016, ApJ, 823, L2

Abbott, B. P., Abbott, R., Abbott, T. D., et al. 2016, Physical Review Letters, 116,061102

Abbott, B. P., Abbott, R., Abbott, T. D., et al. 2016, arXiv:1602.08492

Abbott, B. P., Abbott, R., Abbott, T. D., et al. 2016, ApJ, 818, L22

Abbott, B. P., Abbott, R., Abbott, T. D., et al. 2016, Living Reviews in Relativity, 19,

Bartos, I., Veres, P., Nieto, D., et al. 2014, MNRAS, 443, 738

Berger, E. 2014, ARA\&A, 52, 43

Connaughton, V., Blackburn, L., Briggs, M. S., et al. 2016, American Astronomical Society Meeting Abstracts, 227, 416.02

Dessart, L., Ott, C. D., Burrows, A., Rosswog, S., \& Livne, E. 2009, ApJ, 690,1681

Fairhurst, S. 2011, Classical and Quantum Gravity, 28, 105021

Gou, L.-J., \& Mészáros, P. 2007, ApJ, 668, 392

Horesh, A., Hotokezaka, K., Piran, T., Nakar, E., \& Hancock, P. 2016, ApJ, 819, L22

Jiang, Y.-F., Stone, J. M., \& Davis, S. W. 2014, ApJ, 796, 106

Kiuchi, K., Sekiguchi, Y., Shibata, M., \& Taniguchi, K. 2010, Physical Review Letters, 104, 141101

Klimenko, S., Vedovato, G., Drago, M., et al. 2016, Phys. Rev. D, 93, 042004

Kyutoku, K., Ioka, K., Okawa, H., Shibata, M., \& Taniguchi, K. 2015, Phys. Rev. D, 92, 044028

Levinson, A. 2006, ApJ, 648, 510

Lynch, R., Vitale, S., Essick, R., Katsavounidis, E., \& Robinet, F. 2015, arXiv:1511.05955

Metzger, B. D., Piro, A. L., \& Quataert, E. 2008, MNRAS, 390, 781

Murase, K., Kashiyama, K., Mészáros, P., Shoemaker, I., \& Senno, N. 2016, ApJ, 822, L9
Nakar, E., Ando, S., \& Sari, R. 2009, ApJ, 703, 675

Nakar, E., \& Piran, T. 2011, Nature, 478, 82

Ohsuga, K., Mori, M., Nakamoto, T., \& Mineshige, S. 2005, ApJ, 628, 368

Park, J., Caprioli, D., \& Spitkovsky, A. 2015, Physical Review Letters, 114, 085003

Perna, R., Duffell, P., Cantiello, M., \& MacFadyen, A. I. 2014, ApJ, 781, 119

Rezzolla, L., Baiotti, L., Giacomazzo, B., Link, D., \& Font, J. A. 2010, Classical and Quantum Gravity, 27, 114105

Rosswog, S. 2005, ApJ, 634, 1202

Rosswog, S., Davies, M. B., Thielemann, F.-K., \& Piran, T. 2000, A\&A, 360,171

Rosswog, S., Liebendörfer, M., Thielemann, F.-K., et al. 1999, A\&A, 341, 499

Ruffert, M., \& Janka, H.-T. 2001, A\&A, 380, 544

Sądowski, A., Narayan, R., McKinney, J. C., \& Tchekhovskoy, A. 2014, MNRAS, 439, 503

Sari, R., \& Esin, A. A. 2001, ApJ, 548, 787

Sari, R., \& Mészáros, P. 2000, ApJ, 535, L33

Sari, R., Piran, T., \& Narayan, R. 1998, ApJ, 497, L17

Seto, N., \& Muto, T. 2011, MNRAS, 415, 3824

Thompson, T. A., Chang, P., \& Quataert, E. 2004, ApJ, 611, 380

Veres, P., \& Mészáros, P. 2014, ApJ, 787, 168

Wang, X.-Y., He, H.-N., Li, Z., Wu, X.-F., \& Dai, Z.-G. 2010, ApJ, 712, 1232

Wang, L. J., Dai, Z. G., Liu, L. D., \& Wu, X. F. 2016, ApJ, 823, 15

Yamamoto, T., Shibata, M., \& Taniguchi, K. 2008, Phys. Rev. D, 78, 064054 


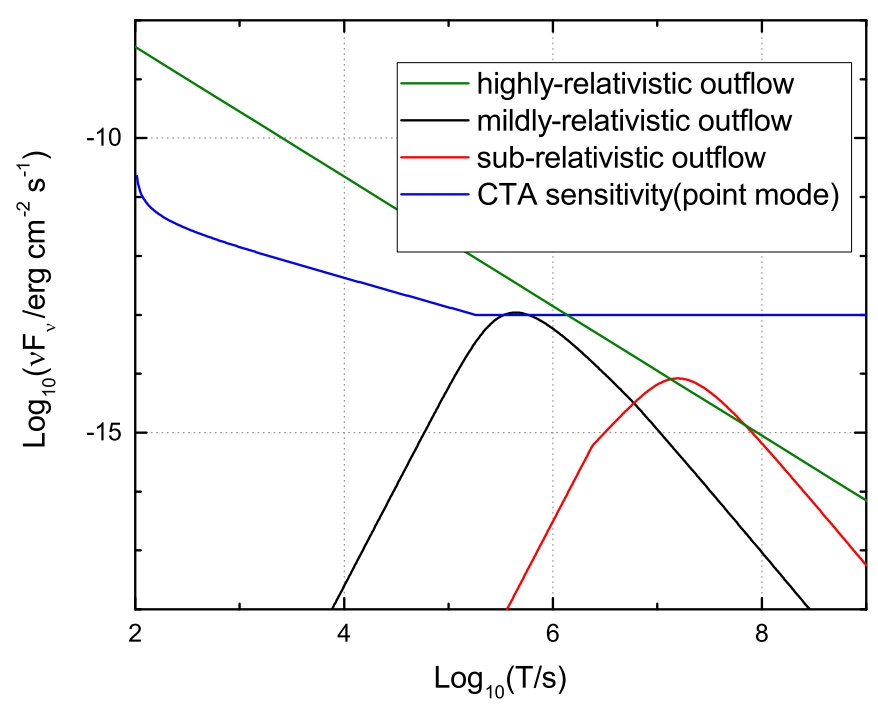

FIG. 1.- TeV light curves in the relativistic outflow scenario (the green line), mildly-relativistic outflow scenario (the black line) and sub-relativistic one (the red line). The green line is calculated with the parameters $E=10^{50} \mathrm{erg}, \Gamma=100$. The black line is calculated with the parameters $E=10^{50} \mathrm{erg}, \Gamma=2$. The red line is calculated with the parameters $E=3 \times 10^{52} \mathrm{erg}, v=0.3 c$. Other parameters used in the calculation are fixed to $\epsilon_{e} / 0.4=\epsilon_{B,-2}=n=D_{L, 26.5}=p / 2.2=1$. The blue line is the CTA sensitivity in the pointing observing mode.
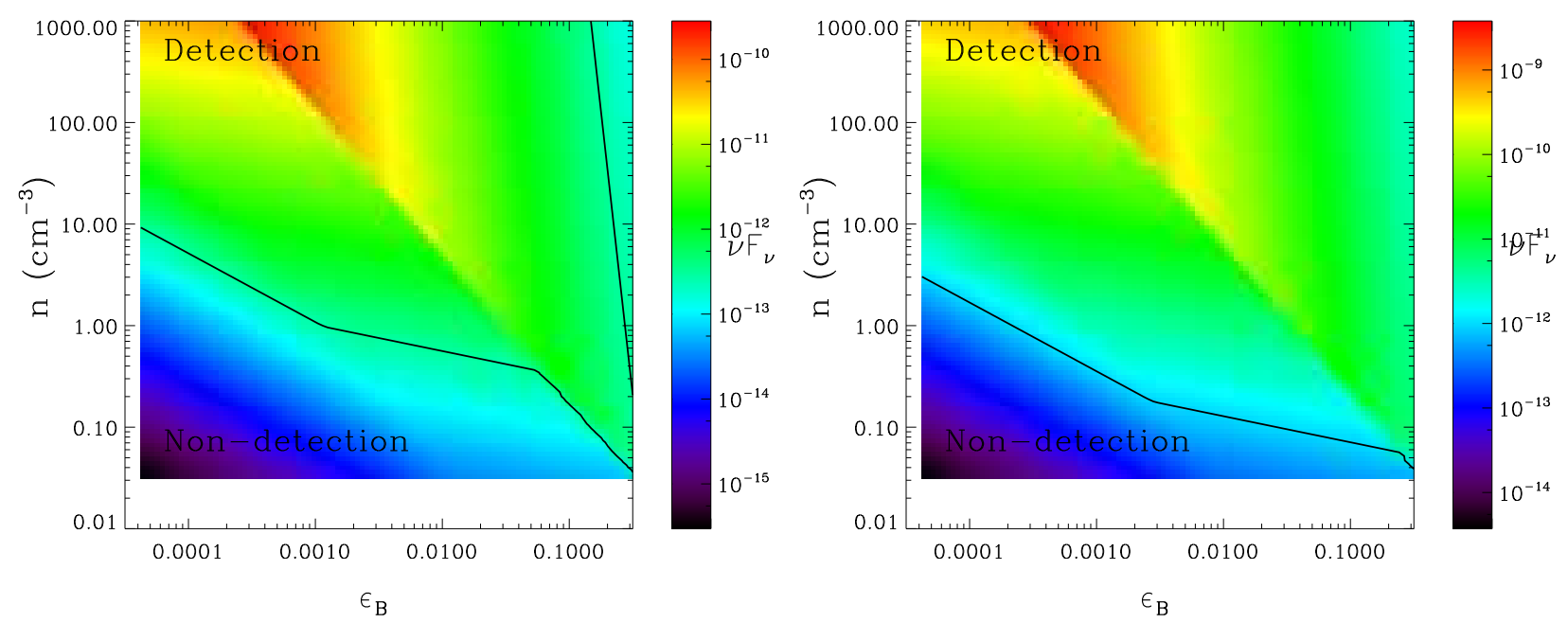

FIG. 2. $-\mathrm{TeV}$ flux in the relativistic outflow scenario. The fluxes are represented by colors in the unit of $\mathrm{erg} \mathrm{cm}^{-2} \mathrm{~s}^{-1}$. We fix $p=2.2, \Gamma=100, \epsilon_{e}=0.4, E=$ $10^{50} \mathrm{erg}$ and $D_{L}=10^{27} \mathrm{~cm}$. The left panel is calculated for $t_{\text {start }}=10^{4} \mathrm{~s}$ and the black line represents CTA sensitivity of 5-hour observation in the point observation mode. The right panel shows the case with $t_{\text {start }}=10^{3} \mathrm{~s}$ and for CTA 0.5 -hour observation sensitivity. 

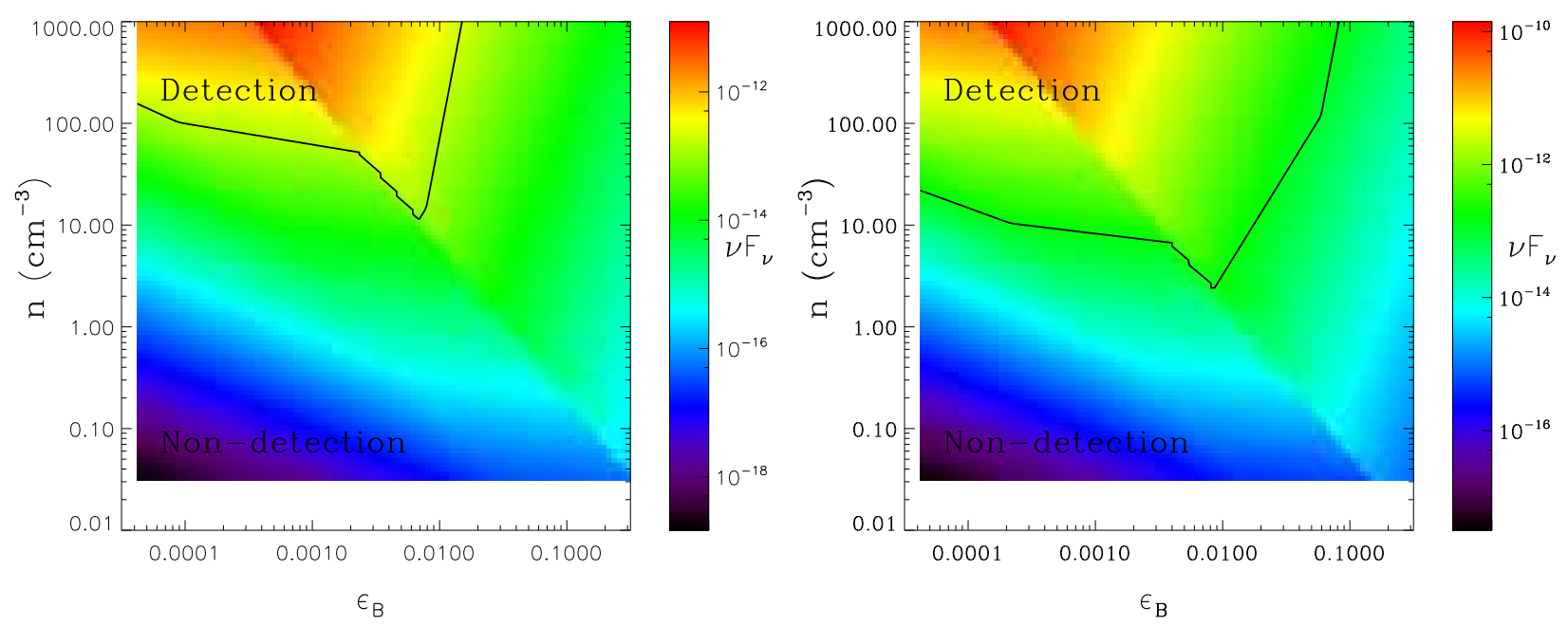

FIG. 3.- TeV flux in the mildly-relativistic outflow scenario for NS-NS mergers (left panel) and NS-BH mergers (right panel). The kinetic energis of the blast waves are assumed to be $E=10^{49} \mathrm{erg}$ and $E=10^{50} \mathrm{erg}$ for NS-NS mergers and NS-BH mergers respectively. Other parameters uased are $p=2.2, \epsilon_{e}=0.4, \Gamma=$ $2, D_{L}=10^{26.5} \mathrm{~cm}$. TeV flux are represented by colors in the unit of erg $\mathrm{cm}^{-2} \mathrm{~s}^{-1}$. The black line shows the sensitivity of CTA 50-hour single-pointing observation.

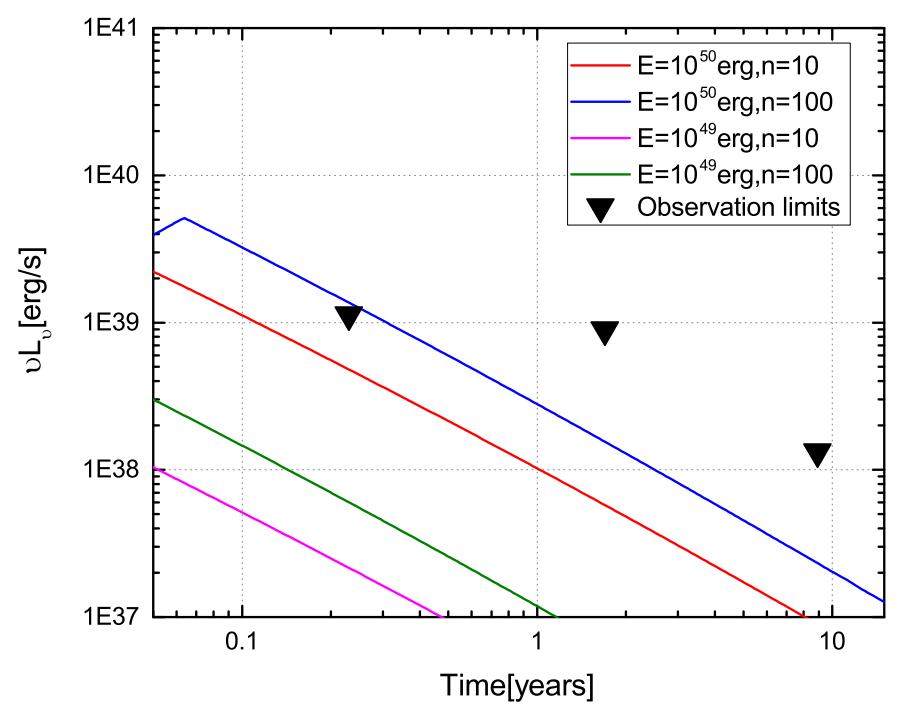

FIG. 4.- Radio emissions at $6.7 \mathrm{GHz}$ from the mildly-relativistic outflow in comparison with the upper limits for GRB 130603B and GRB 060614. Different lines represent different values of number density $n$ and ejecta energy $E$, while other parameters are fixed at $p=2.2, \epsilon_{e}=0.4, \epsilon_{B}=0.01, \Gamma=2$ and $z=0.356$ (the redshift of GRB 130603B). Solid triangles represent the radio upper limits flux taken from Horesh et al. (2016). 